Ks. Franciszek SZULC

(Katowice - Rybnik)

\title{
LA THÉOLOGIE JUDÉO-CHRÉTIENNE DU FILS DE DIEU DANS LE PASTEUR D'HERMAS (SIM. V 2, 1 - 6, 7a)
}

Les nombreuses études consacrées à la christologie du Pasteur d'Hermas laissent jusqu'ici beaucoup de questions sans réponses. Parmi celles les plus fondamentales sont l'origine et la signification du titre de Fils de Dieu dans cette oeuvre. Le texte et le contenu du Pasteur possèdent une longue, comptant un siècle et demi, histoire d'interprétation, couronnée par des travaux récapitulant contemporains ${ }^{1}$. Au centre des intérêtes des chercheurs est la Sim V où l'auteur du Pasteur présenta sa propre conception théologique du Fils de Dieu. Elle était l'objet de nombreuses discussions polémiques au siècle dernier et reste toujours un défi difficile pour les investigateurs de la christologie primitive. Le but des pages qui suivent est d'étudier cette conception théologique d'Hermas en tenant compte des résultats déjà obtenus et de formuler la proposition de son interprétation.

1. Le contexte socio-religieux et historique de l'interprétation. Tout d'abord, il faut bien situer notre problème dans le pluralisme christologique dans le christianisme primitif sans oublier son arrière-plan vétérotestamentaire et judaïque. Avant tout, soulignons-le, c'est la foi en Jésus en tant que Fils de Dieu, déjà présente dans la prédication apostolique, est une pierre de touche de l'identité chrétienne. Et par conséquent, la théologie du Fils de Dieu est devenue le coeur de la question christologique. La théologie biblique du

${ }^{1}$ Cfr. Der Hirt des Hermas. Übersetzt und erklärt von N. Brox, Göttingen 1991; Ph. Henne, La christologie chez Clément de Rome et dans le Pasteur d'Hermas, Paris 1992; idem, L'unité du Pasteur d'Hermas, Paris 1992; R. Joly, Le milieu complexe du „Pasteur d'Hermas”, ANRW II 27/1 (1993) 524-551; J.Ch. Wilson, Five Problems in the Interpretation of the Shepherd of Hermas, Lewiston - New York 1995; M. Leutzsch, Hirt des Hermas, in: U.H.J. Körtner - M. Leutzsch, Papiasfragmente. Hirt des Hermas, Darmstadt 1998, 105-510; A. Schneider, „Propter sanctam Ecclesiam suam". Die Kirche als Geschöpf, Frau und Bau im Bussunterricht des Pastor Hermae, Roma 1999; Shephard of Hermas. A Commentary by C. Osiek, Minneapolis 1999. Nous appuyons nos études sur l'édition critique du texte et la traduction française de R. Joly (Le Pasteur d'Hermas, SCh 53bis, Paris $1968^{2}$ ) en les consultant constamment avec la dernière édition critique du texte de M. Leutzsch, (op. cit.). 
Nouveau Testament nous le montre fort clairement. Un progrès immense des études a permis de connaître plus profondément la genèse et le développement historique de la foi chrétienne au Fils de Dieu². En pénétrant dans ce cercle thématique, quelques auteurs ont consacré leurs études à la christologie judéo-chrétienne ${ }^{3}$. Une analyse détaillée de ces traités nous convainc que nous y avons différents concepts de cette christologie primitive - tant du point de vue méthodologique que de celui du contenu. En respectant ces différences, on peut indiquer des éléments communs qui forment une image rapprochée de la christologie judéo-chrétienne. Parmi les trames thématiques les plus caractéristiques qui fonctionnent déjà en terminologie technique admise, on peut énumérer: la christologie angélique ou angélomorphique (Engelchristologie, angel christology, angelomorphic christology), la pneuma-christologie, la nomos-christologie, l'onoma-christologie. Ce dossier des idées maîtresses n'épuise évidemment pas toute la richesse doctrinale de la christologie dite judéo-chrétienne, ni ne fait connaître des problèmes plus profonds qui tourmentaient les communautés concrètes. Cela se rapporte avant tout au Pasteur où apparaissent tous les thèmes mentionnés ci-dessus. Dans cette oeuvre, qui jouit d'une grande autorité dans l'antiquité chrétienne, surtout en Orient, il y a des expressions particulièrement riches de la foi de l'auteur et de ses coreligionnaires vivant dans un milieu multiculturel à Rome au début du IIe

${ }^{2}$ Dans nos investigations, nous nous basons sur les résultas des auteurs suivants: F. Hahn, Christologische Hoheitstitel. Ihre Geschichte im frühen Christentum, Göttingen 1963 ${ }^{1}, 1974^{4}$ (Gottessohn, p. 280-333); M. Hengel, Der Sohn Gottes. Die Entstehung der Christologie und die jüdisch-hellenistische Religionsgeschichte, Tübingen $1975^{1}$, $1977^{2}$; C. Colpe, Gottessohn, RACh XII 19-88; Anfänge der Christologie. Festschrift F. Hahn, hrsg. C. Breytenbach - H. Paulsen, Göttingen 1991; P.M. Casey, From Jewish Prophet to Gentile God. The Origins and Development of New Testament Christology, Louisville 1991; M. de Jonge, Christologie im Kontekst: die Jesusrezeption des Urchristentums, Neukirchen - Vluyn 1995; M. Hengel, Studies in Early Christology, Edinburgh 1995; M. Karrer, Jesus Christus im Neuen Testament, Göttingen 1998; L.W. Hurtado, Lord Jesus Christ. Devotion to Jesus in Earliest Christianity, Grand Rapids 2003; idem, How on earth did Jesus become a God? Historical Questions abaut earliest Devotion to Jesus, Grand Rapids 2005. Pour l'arrière-fond vétérotestamentaire et judaïque nous nous appuyons sur: H. Cazelles, Le Messie de la Bible. Christologie de l'Ancien Testament, Paris 1978; M. Delcor (éd.), Qumrân, sa piété, sa théologie et son milieu, Gembloux 1978; P. Grelot, Les poèmes du Serviteur. De la lecture critique à l'hermeneutique, Paris 1981; M. Karrer, Der Gesalbte. Die Grundlagen des Christustitels, Göttingen 1990; G.S. Oegema, Der Gesalbte und sein Volk. Untersuchungen zum Konzeptualisiengsprozess der messinischen Erwartungen von dem Makkabäern bis Bar Koziba, Göttingen 1994.

${ }^{3}$ Cfr. J. Daniélou: Théologie du judéo-christianisme, Tournai 1958, 167-218; R.N. Longenecker, The Christology of Early Jewish Christianity, London 1970; A. Vivian, Cristologia dei GiudeoChristiani, „Rivista Biblica Italiana” 22 (1974) 237-256; A. Grillmeier, Jesus der Christus im Glauben der Kirche, Bd 1, Freiburg 1979, $1990^{3}$ (reprint 2004), 138-168; M. Simonetti, Cristologia giudeocristiana: caratteri e limiti, „,Augustinianum” 28 (1988) 51-62; idem, Studi sulla cristologia del II e III secolo, Roma 1993, 7-69; F. Manns, Le judéo-christianisme, mémoire ou prophétie?, Paris 2000, 189-215 (la christologie judéo-chrétienne) 
siècle $^{4}$. Toutes les données concernant la datation et le lieu de sa création concrétisent la méthodologie de nos recherches.

Déjà au point de départ, nous voudrions indiquer les dépendances complexes du sujet ici envisagé. Dans toute communauté, en fonction du milieu socioreligieux et du contexte historique changeant, il apparaissait un problème fondamental: dans quelles catégories conceptuelles et linguistiques doit-on exprimer la foi en Jésus en tant que Fils de Dieu? Les réponses étaient très différenciées dans les communautés judéo-chrétiennes et pagano-chrétiennes ainsi que dans les communautés mixtes et bilingues (Jérusalem, Antioche, Rome). Elles étaient avant tout influencées par deux facteurs: 1. la confrontation avec le paganisme, c'est-à-dire: a) avec les idées mithologiques concernant la divinisation des héros et l'apothéose des gens célèbres, représentées par les

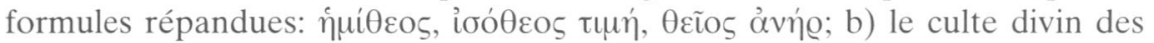
empereurs romains exprimé dans la titulature: divi filius, en Orient: $\theta \varepsilon o v ̃$ víos; 2. les polémiques entre les juifs et les chrétiens ${ }^{5}$. Dans ce contexte, il faut poser quelques questions essentielles envers l'enseignement sur le Fils de Dieu

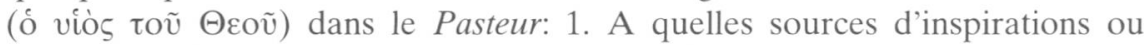
traditions puisa l'auteur?; 2. dans quelle relation reste sa conception du Fils de Dieu par rapport à l'enseignement du Nouveau Testament? 3. Dans quelle mesure le contexte historico-culturel forma-t-il l'enseignement d'Hermas?; 4. Comment peut-on caractériser sa conscience religieuse ainsi que celle de ses coreligionnaires? Toutes ces questions devraient être analysées en tenant compte du caractère différencié de la doctrine du christianisme primitif entre la fin du I ${ }^{\mathrm{er}}$ et la moitié du $\mathrm{II}^{\mathrm{e}}$ siècle.

\section{Les schémas d'interprétation sur la base de la pneuma-christologie.} Depuis très longtemps, prédominait l'opinion qu'Hermas était un représen-

${ }^{4}$ Cfr. P. Lampe, Die stadtrömischen Christen in den ersten beiden Jahrhunderten. Untersuchungen zur Sozialgeschichte, Tübingen $1989^{2}$; M. Leutzsch, Die Wahrnehmung sozialer Wirklichkeit im „Hirten des Hermas”, Göttingen 1989; Schneider, op. cit., p. 395-483.

5 Nous disposons actuellement d'un dossier riche concernant ces deux problèmes: I. Leipoldt - W. Grundmann, Umwelt des Urchristententums, Bd 1, Berlin 1973³, 127-142 (Herrschekult in Rom, p. 138-142); Bd 2, $1972^{3}$, p. 102-113; Colpe, op. cit., p. 27-41; F. Manns, L'Isral de Dieu. Essais sur christianisme primitif, Jérusalem 1996, 219-302; idem, Le judéo-christianisme, mémoire ou prophétie?, p. 63-93; C.J. Setzer, Jewish Responses to Early Christians. History and Polemics, Minneapolis 1994, 30-150; T. Kriener, „Glauben an Jesus” - ein Vertoss gegen das zweite Gebot? Die johanneische Christologie und der jüdische Vorwurf des Götzendienstes, Neukirchen - Vluyn 2001; Hurtado, How on Earth Did Jesus Become a God?, p. 152-178 (Early Jewish Opposition to Jesus-Devotion). Il faut analyser ce problème à l'intérieur du processus de séparation du judaïsme et du christianisme aux $\mathrm{I}^{\mathrm{er}}$ et $\mathrm{II}^{\text {ième }}$ siècles. Ce phénomène socio-religieux complexe est profondément traité par: J.D.G. Dunn (éd.), Jews and Christians. The Parting of Ways A.D. 70 to 135, Tübingen 1992; B. Wander, Trennungsprozesse zwischen Frühen Christentum und Judentum im 1. Jahrhundert n. Chr., Tübingen 1994; S.G. Wilson, Related Strangers. Jews and Christians, 70-170 C.E., Minneapolis 1995 
tant de la pneuma-christologie qui fut née tout au début du christianisme et qui

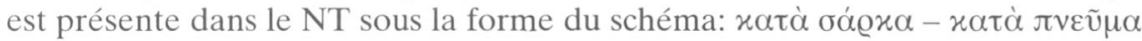
(voir $\mathrm{Rm} \mathrm{1,3-4).} \mathrm{L'évolution} \mathrm{ultérieure} \mathrm{de} \mathrm{ce} \mathrm{type} \mathrm{de} \mathrm{christologie,} \mathrm{appelée}$ aussi la christologie pneumatologique (ou pneumatique), fut traitée par des auteurs très connus dans le cadre de l'histoire du dogme: A. von Harnack ${ }^{6}$, R. Seeberg ${ }^{7}$, F. Loofs $^{8}$, A. Grillmeier ${ }^{9}$, M. Simonetti ${ }^{10}$. Dans leurs ouvrages, considérés déjà comme classiques, ils exposèrent aussi leurs idées au sujet de la pneuma - christologie dans le Pasteur. Nous avons envisagé les opinions de ces chercheurs au point de départ de nos études.

Cependant nous consultons et confrontons constamment nos propres recherches surtout avec les résultats obtenus par ces chercheurs qui analysèrent directement et plus profondément la problématique de la christologie dans la Sim

${ }^{6}$ Cfr. Lehrbuch der Dogmengeschichte, Tbingen 1886-1890, 1909 (reprint: Darmstadt 1964), Bd 1, 207-212 (Hermas, p. 211-212); c'est cet auteur qui a introduit les termes techniques: „Geistchristologie” et „pneumatische christologie” qui fonctionnent jusqu'à présent dans l'histoire des dogmes.

${ }^{7}$ Cfr. Lehrbuch der Dogmengeschichte, Leipzig 1895, $1920^{3}$ (reprint: Darmstadt 1974), Bd 1, 212-218.

${ }^{8}$ Cfr. Leitfaden zum Studium der Dogmengechichte, Halle 1889; 19596 $1968^{7}$ (ergänz. und hrsg. von K. Aland), p. 63-72. Les sources riches qu'il a rassemblées concernant largement Geistchristologie ont été publiées après sa mort: F. Loofs, Theophilus von Antiochen adversus Marcionem und die anderen theologischen Quellen bei Irenaeus, Leipzig 1930.

9 Cfr. Jesus der Christus im Glauben der Kirche, p. 146-149, 157-160. La caractéristique du Pasteur d'Hermas faite par cet auteur exige d'être revisée. D'un côté, cet écrit a été qualifié avec raison au courant de la christologie judéo-chrétienne (voir Das alte Erbe - das Christusbild der jüdisch-christliche Theologie, p. 138-152) dans le cadre de Motive archaischer Christologie. De l'autre, l'ensemble de la Sim V a été placé à part, dans un recueil de trames intitulé: Das volkstümliche Christusbild (p. 158-160). Cette qualification est fort contestable et déprécie la valeur de la théologie d'Hermas. Les spéculations théologiques raffinées dans la Sim V ne peuvent être dans un aucun cas un fruit de la création populaire. D'où, l'interprétation de Grillmeier de la Sim V devrait être corrigée et complétée.

${ }^{10}$ Cfr. Note di cristologia pneumatica, ,Augustinianum” 12 (1972) 201-232; le reprint avec de petites modifications in: idem, Studi sulla cristologia del II e III secolo, Roma 1993, p. 23-51 (Cristologia pneumatica). D’abord, nous présentons sa définition de la christologie pneumatique: „Col nome di cristologia pneumatica e di Geistchristologie indichiamo l'antica concezione cristologica che definisce come spirito (pneuma) la componenta divina di Gesu, cioe il Cristo preesistente", voir ibidem, p. 201 (= Studi sulla cristologia, p. 23). Dans la systématisation de cette théologie, il a admis la division suivante: ,una fondamentale tripartizioni: alcune volte il termine pneuma, in riferimo a Cristo, indica la sua natura divina; altre volte indica la persona del Cristo preesistente; altre volte in questo senso giunge ad indentificare il Cristo preesistente con lo Spirito santo", cfr. ibidem, p. 203 (= Studi sulla cristologia, p. 25). Il est contestable que ces définitions soient justes par rapport au développement précoce de cette christologie et surtout l'on ne peut les appliquer au Pasteur. Mais le dossier recueilli par Simonetti sur la christologie pneumatologique dans l'antiquité chrétienne est très précieux pour les études suivantes de cette problématique, entre autres sous aspect de l'orthodoxie de cette christologie: voir Geistchristologie e binitarismo, voir ibidem, p. 226-232 (= Studi sulla cristologia, p. 46-51). 


\section{Nous les citons dans l'ordre chronologique: M. Dibelius ${ }^{11}$, J. N. D. Kelly ${ }^{12}$, L. Pernveden ${ }^{13}$, L. Cirillo ${ }^{14}$, L. W. Nijendijk ${ }^{15}$, M. Leutzsch ${ }^{16}, \mathrm{Ph}_{\text {. Henne }}{ }^{17}$,}

${ }^{11}$ Dans son commentaire Hirt des Hermas, Tübingen 1923, il a donné sa propre interprétation de la Sim V. Par rapport au texte qui éveille le plus de controverses, soit Sim V 6,5, il a admis que: „In Wirklichkeit liegt eine ganz neue Allegorie vor: Gegenstand der Schilderung in 5 ist nicht mehr

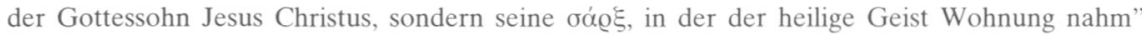
(ibidem, p. 571). Il a interprété l'essentiel de la christologie du Pasteur d'une manière suivante: „Das in der $\sigma \alpha \dot{\varrho} \varrho \xi$ Christi wohnende $\pi v \varepsilon \tilde{v} \mu \alpha$ wird deutlich beschrieben als vorweltliches Schöpfungsprinzip s. auch Sim. IX 12, 2. Dieser Geist ist es, der die Werke Christi getan hat; die oó@ $\xi$ hat nur mitgewirkt. Dieser Geist wird nun 6, 4. 7 auch als „Sohn Gottes” bezeichnet; das ist vielleicht durch die Figur des Sohnes im Gleichnis angeregt, aber in 6, 7 keinesfalls allein von dorther bedingt. Vollends beweist Sim. IX 1, 1, dass Hermas den heiligen Geist mit de Sohn Gottes, d. h. mit dem präexistenten Christus identifiziert" (ibidem, p. 574). A la lumière des études ultérieures cette interprétation est inacceptable.

12 Cfr. J.N.D. Kelly, Early Christian Doctrins, London 1960, 1977²: l'auteur écrit de „Spirit or Pneumatic Christolgy” (ibidem, p. 142-144), et quant au Pasteur, il exprime „Hermas theology was thus an amalgam of binitarnism and adoptionism".

13 Il faut noter ici que c'est L. Pernveden qui a initié des études méthodologiquement mieux fondées. Dans son livre The Concept of the Church in the Shepherd of Hermas (Lund 1966), il a consacré beaucoup de place à la problèmatique christologique (Hermas Christological concepts, p. 38-71; The Son of God and the Church, p. 72-111). C'est lui qui a indiqué des insipirations de Is 42,1 dans le texte d'Hermas: „It is not impossible that there are ideas from Is. 42 interwoven in this particular Christological concept. The concept of the one sent from God as a servant is a basic idea in both. In both cases the servant is described as being chosen; it seems too as if in both cases the servant is in some way pleasing to his master. The Spirit is given to both of these servant figures. Both are apparently appointed to fulfil the judgement of God (in Hermas this is expressed more indirectly), and both are intermediaries of the law" (ibidem, p. 47). Il a aussi donné une interprétation juste du titre de Fils de Dieu et a justifié qu'il ne faut pas identifier le Fils de Dieu à l'EspritSaint: „The conclusion then seems to be that it is through the link with the Spirit that the chosen flesh comes to be called the Son of God. Or in other words the concept Son of God indicates this chosen flesh, when, in obedience to the divine command, through the strength of the Spirit, in the service of the same Spirit, and together with the Spirit it carries out the act of salvation [...]. In Sim. $\mathrm{V}$ the Spirit is not described as the Son of God but only as the son, and the son relationship is only present in the text of the parable itself, not in the explanation [...]. We must therefore differentiate between the term son on the one and the term Son of God on the other. Son of God is not a genetic term, but it is to be taken in connection with obedience and the act of salvation. The term son has a more genetic implication, albeit in a spec way" (ibidem, p. 50).

${ }^{14}$ Cfr. La christologie pneumatique de la cinquieme parabole du „Pasteur” d'Hermas, RHR 93 (1973) 25-48. Parmi tous les auteurs, c'est lui qui a approfondi les études sur la Sim V et a obtenu les résultats les plus justifiés dont nous profitons ici.

15 Cfr. Die Christologie des Hirten des Hermas (diss.), Utrecht 1986, 83-97. Nous ne pouvons pas accepter les résultats obtenus par cet auteur, voir les critiques de N. Brox, op. cit., p. 306, 327 , 364, 378, 486, 491, 494.

${ }^{16}$ Cfr. M. Leutzsch, Die Wahrnehmung sozialer Wirklichkeit im „Hirten des Hermas”, op. cit., p. $144-153$.

17 Cfr. A propos de la christologie du Pasteur d'Hermas. La cohérance interne des niveaux d'explication dans la Cinquième Similitude, RSPT 72 (1988) 569-578; idem, La véritable christologie de la Cinquième Similitude du „Pasteur” d'Hermas, RSPT 74 (1990) 182-204; idem, La christologie chez Clément de Rome et dans le Pasteur d'Hermas, Paris 1992, 157-210. Voici ses propositions 


\section{N. Brox ${ }^{18}$, R. J. Hauck ${ }^{19}$, A. Stewart-Sykes ${ }^{20}$, C. Osiek ${ }^{21}$. Ici, nous ne pouvons ni les présenter pleinement, ni discuter avec eux. Nous le faisons dans notre livre sur Le Fils de Dieu dans le «Pasteur» d'Hermas ${ }^{22}$.}

méthodologiqes: „C'est ainsi que nous avons pu élaborer la théorie de la cohérence interne des niveaux d'explication. L'idée de base de cette théorie est fort simple: le Pasteur explique le recit porabolique selon trois perspectives biens définies: la pratique du jeûne (H Sim V 5, 1 - 6, 3), la christologie (H Sim V 5, 1 - 6, 3) et la soteriologie (H Sim V 6, 4-7,4)" (La véritable christologie, p. 182. L'auteur a appliqué cette directive dans son livre: La christologie chez Clément de Rome, p. 172-183. Nous n'acceptons pas cette méthodologie et la division du texte ici présenté. Avant tout, nous faisons de l'opposition à la thèse principale de l'auteur: „Sim V 6, 4 - 8 n'est pas christologique, parce qu'il n'y a pas aucune mention du Fils de Dieu, ni expilcite, ni implicite: Son nom n'est pas mentionné ; aucune allusion n'est faite à Son action telle qu'elle fut décrite dans la parabole ou dans son interprétation christologique" (La christologie chez Clément de Rome, p. 174).

${ }^{18}$ Cfr. Hirt des Hermas, p. 33, 306, 320-322. Sa critique de Ph. Henne est d'après nous juste: „Die von Henne, A propos de la christologie, 569-578 endeckte konzentrische Konstruktion der Sim V, in der zwei Dialoge über Moral eine Parabelerzählung und ihre Erklärungen einrahmen, ist in ihrer Künstlichkeit nicht die Sache des H." (ibidem, p. 307, note 17); et quant à l'interprétion: „Henne, A propos de la christologie, 569-578 macht den gravierenden Fehler, den redaktionellen Beitrag des $\mathrm{H}$ im Gebrauch seiner Stoffe (hier: „Fleisch und Geist”) nicht einzukalkulieren und den Passus 6, 4-7 (trotz 5, 1 - 6,3) nicht mehr christologisch zu lesen, sondern auf den heilsrelevanten Bewährungsprozess des Menschen generell zu beziehen” (ibidem, p. 320).

19 Cfr. R.J. Hauck, The Great Fast: Christology in the Shepard of Hermas, ,Anglican Theological Review" 75 (1993) 187-188. L'auteur essaie d'expliquer le contenu de la Sim V du point de vue du jeûne et de $\delta ı \psi \iota x i \alpha$. A son avis, la figure du Fils de Dieu possède un sens exclusivement sotériologique en tant que modèle pour tous les croyants de la coopération parfaite avec le SaintEsprit. Remarquons que c'est une problèmatique dérivative et secondaire, et l'auteur ne touche pas aux problèmes fondamentaux. L'auteur a posé des questions faciles à l'adresse d'Hermas et c'est pourquoi il a réçu des réponses semblables.

${ }^{20}$ Cfr. The Christology of Hermas and the Interpretation of the fifth Similitude, „Augustinianum" 37 (1997) 273-284. L'auteur présente une revue riche de diverses interprétations de la Sim V. Il a accentué avec raison surtout deux questions que nous soulevons dans la suite de cet article. En premier lieu, nous allons répondre à la question suivante: „Hermas has introduced servant christology into this contekst, and has at the same time constructed a spirit christology. The reasons for doing this are not clear, but given Hermas' overall ethical interest it may be moral" (ibidem, p. 281). La seconde question concerne la qualification doctrinale: „Whereas we may agree with Kelly and Gieschen in continuing to attribut a Spirit Christology to Hermas, whether this might properly be called binitarian or adoptionist is less clear. The problem with these terms is that they are generally used in a derogatory sense" (ibidem, p. 283).

${ }^{21}$ Cfr. Rich and poor in the Shepherd of Hermas, op. cit., p. 168-183. Son commentaire évoque une déception terrible dans plusieurs points. Nous devons ici nous limiter à ses trois opinions fort contenstables. Déjà dans l'Introduction, elle donne une image caricaturale du contenu de la Sim V: „In the parable of the Fifth Similitude and its interpretation, there is an awareness of a triad of Father, Spirit, and Son. They are distinct characters with quite different roles to play, slave/adopted son versus full son of the God-figure. Yet, according to the parable, the Holy Spirit is God's original son, the one later called Son of God, the son subsequently becoming the Spirit's brother by an act of God" (Shephard of Hermas, p. 36); ,Yet, though v. 4b implies that the same interpretation is continuing, in fact these verses have moved off into something different with not much by way of transition. It is likely that they speak of Christ as primary referent, but, as usual in Hermas, only for the sake of moral instruction and paraenesis, to which the consideration quickly moves. Thus, the 
Le problème fondamental et à la fois le plus important, qui ne fut pas suffisamment expliqué dans les études mentionnées, est la genèse et le caractère doctrinal de la conception théologique du Fils de Dieu qu'Hermas développa uniquement dans cette partie de son écrit. Cela implique quelques questions particulières, aussi considérables, qui restaient jusqu'à présent sans réponse: 1. pourquoi le Saint-Esprit fut-il appelé le Fils (ó viós)?; 2. de quelles sources d'inspirations profita l'auteur?; 3. qui et en quel sens a-t-il été nommé le Fils de Dieu (ó viòs toṽ @eoṽ)?; 4. est-ce qu'il existe des liens entre sa conception théologique et les écrits néotestamentaires? 5. quelle est la tradition religieuse et théologique à laquelle l'auteur appartient et quelles innovations introduisit-il à l'articulation de la foi au Fils de Dieu?

\section{Les principes fondamentaux de l'interprétation de la Sim V. Avant de} traiter l'ensemble des questions mentionnées ci-dessus, nous avons fait une analyse profonde de la structure et du contenu de la Cinquième Similitude. Nous avons démontré que la parabole du vignoble (Sim V 2,1-11) joue dans le texte un double rôle: 1 . mise dans le contexte des instructions sur le jeûne (Sim V 1, 1-5 et Sim V 3,1-9), elle fournit un exemple édifiant d'un service fervent d'un „esclave très fidèle” comme un modèle à suivre pour „les serviteurs de Dieu"; ici, il est nécessaire de préciser qu'en dépit des opinions propagées pendant longtemps, comme M. Leutzsch ${ }^{23}$ l'a prouvé, la parabole ne

humanity of Christ becomes the bridge to a third interpretation of the parable-one that departs substantially from the original story line” (Shephard of Hermas, p. 180); ,Yet the strictly christological perspective can be said to be adoptionist, since there is no indication of preexistence of the Son of God, and perhaps binitarian, since God and preexistent spirit belong to the same family. But the theology here is more accurately monotheistic with preexistent (but not necessarily divine) spirit and exalted Son of God" (Shephard of Hermas, p. 181).

22 Cfr. F. Szulc, Syn Boży w Pasterzu Hermasa. Swiadectwo chrystologii judeochrzescijańskiej, SACh NS 2), Katowice 2006 (en polonais avec un résumé en français, p. 190-198). Dans la suite de cet article, nous présentons les résultats les plus importants contenus dans le livre cité ci-dessus (voir, p. 114-151). Ici, nous avons accentué les conculsions et postulats découlant de ces recherches. Avant tout, nous espérons enrichir le savoir sur le judéo-christianisme dont nous nous occupons depuis longtemps: F. Szulc, Struktura teologii judeochrześcijańskiej. Studium metodologiczne w świetle badań J. Daniélou, Lublin $1982^{1}$, Kraków $2005^{2}$ (en polonais), les résultats obtenus ont été publiés en français dans l'article: La théologie judéo-chrétienne: l'actualité de la conception de Jean Daniélou et l'essai de son développement, VoxP 24 (2004) t. 46-47, 423-432.

${ }^{23}$ Quant à la provenance des éléments pricipaux du contenu de la parabole, et surtout du motif de „l'esclave très fidèle”, ce chercheur constate: „Die Befremdlichkeit des Gleichnisses erwiesen sich mithin als scheinbar, wenn man durchweg nicht jüdische (wie Dibelius, Hirt, s. 565) sondern römische Verhältnisse als adäquaten Verstehenshintergrund von Sim V 2 namhaft macht. Weder sind die «Motive» des Weinbergs, des Unkrauts und der Abreise des Herrn ausschließlich jüdisch noch ist es die Belohnung aufgrund überschüssiger Werke (gegen Dibelius, ebd.) [...]. Das Gleichnis als Ganzes beschreibt einen Einzelfall sozialen Aufstiegs. Es ist damit auf dem Hintergrund der Aufstiegsmöglichkeiten der zeitgenössischen römischen Gesellschaft plausible [...]" (Die Wahrnehmung sozialer Wirklichkeit im „Hirten des Hermas”, p. 150). 
possède pas de provenance biblique - ni juive, ni chrétienne - mais elle reproduit d'une manière fidèle le processus de libération et d'adoption de l'esclave dans la réalité sociale et légale de l'Empire romain; 2. pourtant, la parabole constitue avant tout, selon la conception de l'auteur, le point de départ pour la présentation de sa propre théologie du Fils de Dieu, elle fut également conçue comme la préparation du lecteur à la réception de cet enseignement; il faut ici souligner le fait que bien que l'auteur se réfère dans ses spéculations, dites explications, à la parabole, en réalité les sources dont il tire ses arguments se trouvent hors d'elle et il faut seulement les découvrir.

Les vraies idées sur le Fils de Dieu sont exprimées dans trois explications de la parabole: 1. Sim V 5, 1-4; 2. Sim V 6, 1-4a; 3. Sim V 6, 4b-7a. Par „l'ex-

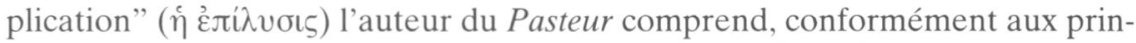
cipes apocalyptiques de sa pensée, la présentation de la vérité plus profonde et à la fois cachée dans la parabole. Et par conséquent, nous disposons alors de trois „révélations” dont chacune possède ses propres sources d'inspirations. $\mathrm{Au}$ début de la première explication il y a déjà un énoncé surprenant: „Le fils, c'est

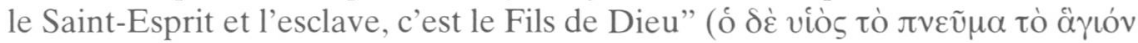

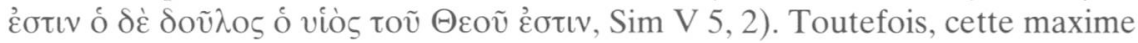
préliminaire explicative n'a son véritable sens qu'après y avoir introduit le contenu inclu dans deux explications suivantes de l'auteur. Nous avons démontré qu'en ce cas cette phrase prend forme: le Saint-Esprit, c'est le Fils et le Serviteur, c'est le Fils de Dieu. Dans cette forme, elle exprime d'une manière fidèle la conception théologique d'Hermas et est en même temps la synthèse et l'essentiel de sa pensée. Cependant cette déclaration doctrinale capitale exige une argumentation approfondie sans laquelle elle reste incompréhensible et étrangère par rapport à la tradition tant judaïque que chrétienne. Et c'est cela qui constitue la difficulté principale et une source de nombreuses querelles parmi les chercheurs car l'auteur même ne justifia pas d'une manière claire et suffisante le contenu de la maxime prononcée.

Par suite de l'analyse d'une longue discussion scientifique sur ce sujet dans la Sim V, nous avons tiré une conclusion suivante du caractère méthodologique: puisque la parabole du vignoble ne constitue pas une source d'arguments théologiques de l'auteur, il faut les chercher dans les trois explications, mais de sorte à parvenir aux principes profonds y contenus ainsi qu'aux idées théologiques y présentes implicite, c'est-à-dire dans les traditions sous-jacentes du texte. Voici nos résultats obtenus.

Le principe fondamental de la théologie d'Hermas est un monothéisme strict, exprimé plusieurs fois et bien souligné dans les formules solennelles de foi, typiques pour le judaïsme de la diaspora (voir Mand I 1-2) C'est sur ce fondement que l'auteur appuya les principes anthropologiques et pneumatologiques qui sont très rapprochés du point de vue doctrinal des croyances de la communauté de Qumrân, et il est fort probable qu'ils en tirent ses origines. Il y 
a deux faits constatés par nous qui en sont la preuve: 1 . le schéma dualiste et le contenu des instructions morales dans les Préceptes (voir 1 QS, 3, 13-4,26); 2. la doctrine du Saint-Esprit est très proche de l'enseignement inclu dans les textes qumrâniens: il existe une conviction commune que l'Esprit fut déjà donné et est présent dans toute la communauté (1 QS 3, 7-9; 1 QH 7, 6-7; 14,3) ainsi qu'en chacun (1 QH 4, 31-32; 7, 6-7; 14, 11-13). Il est très siginificatif que les membres de la communauté de Qumrân sont nommés comme „choisis”, ,justes” et „saints” et c'est ainsi qu'Hermas désigne ses coreligionnaires. Les principes y montrés constituent les fondements de la conception de la „,chair choisie” dans laquelle „l'Esprit Saint prit demeure”, et cette conception servit ensuite à expliquer, comme l'auteur même le détermina, la question de «l'héritage à

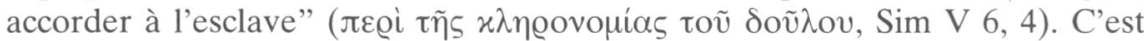
juste autour de cet ,héritage” que se concentrent tous les énoncés dans le texte. Dans la parabole, ,l'esclave très fidèle” fut libéré et devint le cohéritier. Il nous reste à chercher quel héritage réçut „la chair choisie" et qui se cache sous cette formulation.

4. Le Fils de Dieu à la lumière de la théologie judéo-chrétienne. Après avoir étudié l'ensemble des spéculations au premier regard énigmatiques dans la Sim V, riches en métaphores et allusions, nous sommes arrivés à la conclusion que l'auteur revèle, par la bouche du Pasteur que la prophétie concernant le Serviteur de Yahvé fut accomplie et que dès lors il a le droit à la dignité et au titre de Fils de Dieu. Autour de cette vérité sont concentrées toutes les démarches d'interprétation de l'auteur qui essaie de justifier théologiquement cette vérité et d'y attribuer sa valeur voulue en vue des destinataires ainsi que de la situation socio-religieuse compliquée. La reconstruction de son argumentation a permis de découvrir qu'elle se compose de plusieurs éléments d'une différente provenance.

A l'étape préparatoire, c'est-à-dire dans la parabole du vignoble, l'auteur introduisit de telles figures qui étaient conçues par avance comme des prototypes de ceux personnages qui devaient apparaître dans les explications. Ensuite, ces personnages, après les avoir transportés (la première explication) du monde de la culture romaine et hellénistique au mode de notions véterotestamentaires et judaïques, changèrent radicalement leur valeur. L'auteur trouva les plus grandes difficultés dans le processus de traduction de la relation (de la comparaison) esclave - Fils de Dieu, ce qui dévoilent deux explications suivantes consacrées à ce problème. Cependant le fait que la figure du fils Esprit Saint n'exigeait pas d'explications est significatif.

Le processus d'explications - révélations à l'intérieur de la Sim V s'effectue dans le dialogue entre Hermas et le Pasteur. Le premier pose des questions difficiles au nom du lecteur, et le deuxième remplit la fonction de porte parole de l'auteur. Les déclarations du Pasteur trahissent différentes sources d'inspi- 
rations de l'auteur ainsi que ses propres interprétations. Il s'inspire de la Bible hébraïque et des traditions juives consciennement choisies en omettant les idées capitales du judaïsme hellénistique. Le tout est enraciné dans les structures de la pensée hébraïque. Elles manifestent en somme la théologie du Fils de Dieu d'Hermas dont les éléments et la structure nous présentons ici brièvement: 1. La conception de „la chair choisie dans laquelle l'Esprit Saint prit demeure" (Sim V 6, 5) s'appuie sur la prophétie de Is 42, 1, dont le contenu fut interprété comme réalisé à la base de la foi de l'auteur et approfondi par ses

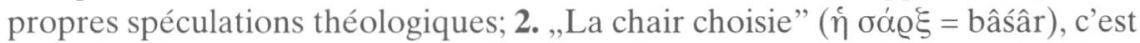

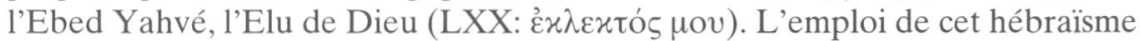
indique l'omission intentionnelle de la Septante qui préfère l'interprétation collective et où Ebed - Serviteur est traduit le plus souvent comme $\pi \alpha \tilde{\zeta} \varsigma$

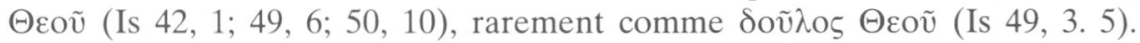

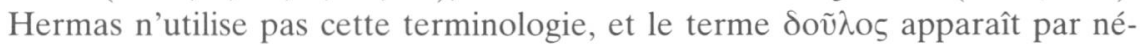
cessité dans les comparaisons, toutefois ,le Fils de Dieu n'apparaît pas sous la forme d'un esclave" ( Cela manifeste aussi une opposition de l'auteur par rapport à l'utilisation pour

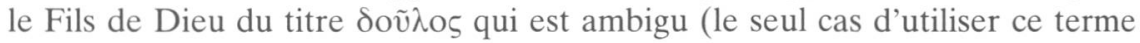
dans $\mathrm{Ph} .2,7$ est justifié du point de vue théologique et linguistique pour exprimer la kénose du Christ). Sa pensée théologique se développe indépendamment de la christologie du Nouveau Testament (voir $\pi \alpha \tilde{\iota} \varsigma$ - christologie: Mt 12,18; Ac 3, 13.26; 4, 27.30; 1 Clém 59, 2; Did 9, 2; Barn 9, 2); 3. L'Esprit Saint, c'est Ruah Yahvé - rûah haqqōdeš qui non seulement „reposa” sur le Serviteur mais également „prit demeure” en Lui. La doctrine du Saint-Esprit possède une provenance vétero-testamentaire et judaïque avec une forte nuance qumranienne, et la terminologie fut empruntée au judaïsme hellénistique

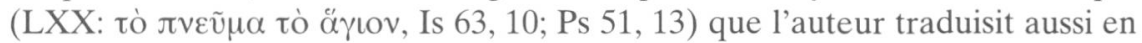

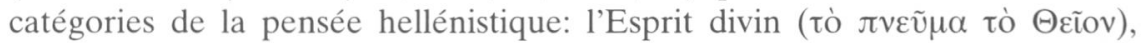
l'Esprit de la divinité ( dignité divine du Fils de Dieu fut consolidée. En revanche, l'auteur puisa l'idée de Fils - Esprit Saint aux spéculations rabbiniques au sujet de be'rešit (ce qui signifie: „commencement”, ,fils”) dans Gen 1, 1, qui étaient connues aussi par les chrétiens; 4. Par le fait d"' avoir pris demeure" du Fils - Esprit Saint dans ,la chair choisie", donc en Serviteur de Dieu (en effet en Jésus), il fut élevé à la dignité du Fils de Dieu et dès lors ce n'est que Lui qui a le droit à ce titre; 5. La dignité de Fils de Dieu et sa mission salvifique furent exprimées d'une manière éminente dans la Sim V 6, 2-3. Dans ce texte, il y a une petite somme de la théologie du Fils de Dieu d'Hermas. A sa lumière: „le Fils de Dieu n'apparaît pas sous la forme d'un esclave, mais avec grande puissance et souveraineté (عí

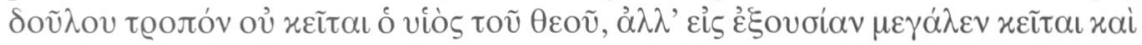

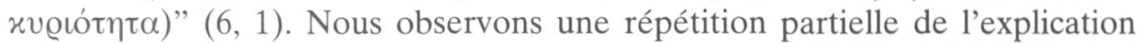
précédente de la parabole du vignoble mais avec un accent plus fort sur le 
fait que Dieu a confié son peuple à son Fils qui ,a purifié leurs péchés” (xai

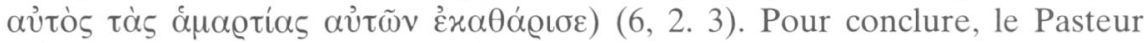
affirme: „Tu vois, dit-il, qu'il est le Seigneur de son peuple, puisqu'il a reçu

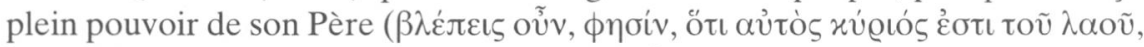

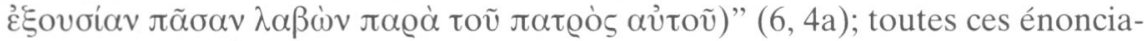
tions sont enracinés dans la prédication apostolique.

En résumant, la conception théologique du Fils de Dieu dans la Sim V se distingue par une grande richesse doctrinale et par une originalité. L'auteur joignit dans sa conception d'une manière créative la foi monothéiste avec la foi en Serviteur de Dieu - Jésus, en qui Dieu réalisa ses promesses de salut envers son peuple. C'est Lui qui reçut de la part de Dieu, par le fait d'avoir pris en Lui demeure de l'Esprit Saint - de l'Esprit de la divinité, la dignité et le titre de Fils de Dieu. Mais conformément à ce jugement, on ne peut pas dire que le SaintEsprit „s'incarna” en Jésus car l'idée d'incarnation fut étrangère à la mentalité hébraïque. A la lumière de tout l'enseignement concernant le Fils de Dieu dans la $\operatorname{Sim} \mathrm{V}$, qui a été ici brièvement présenté, nous pouvons constater qu'il possède un caractère proprement judéo-chrétien.

5. Conclusions et postulats. L'enseignement d'Hermas concernant le Fils de Dieu ne donne pas de raisons à reprocher l'apothéose de l'homme, un tel reproche apparaissait souvent dans les polémiques entre les juifs et les chrétiens (voir Jn 7; 10, 21 - 39) et il était sûrement vif dans sa communauté romaine où était répandu le culte divin des empereurs. Les spéculations théologiques d'Hermas n'ont rien de commun avec les fils divins de la mythologie, même le vocabulaire utilisé ne dicte pas de telles associations. D'autre part, il élimina la messianologie et, d'une manière conséquente, le mot Christ n'apparaît jamais dans son oeuvre. Les raisons d'une telle décision de l'auteur du Pasteur sont, d'après nous, les suivantes: 1 . le titre messianique réveilla des controverses avec les Juifs; 2. pour les pouvoirs romains, il eut un caractère provocatif et put causer des persécutions; 3. pour les païens, appelés à la conversion, il fut complétement incompris; grâce à l'onction pratiquée dans l'Antiquité, l'homme entrait dans le panthéon des dieux. Mais pour les raisons théologiques, Hermas n'eut pas besoin du terme Christ et de la notion de Messie afin d'élaborer sa théologie du Fils de Dieu. D'où dans les interprétations contemporaines du Pasteur, l'on ne peut introduire ce terme car dans ce cas-là, cela entraîne déjà au point de départ une déformation de sa théologie. Malheureusement, c'est une pratique fréquente dans les études sur „la christologie" du Pasteur. Par conséquent, de nombreuses ,interprétations christologiques", peu ou mal précisées comme nous l'avons démontré, conduisent à des malentendus insurmontables. Nous ne pouvons pas non plus prendre sa doctrine pour l'adoptionisme car cette catégorie théorétique (plutôt le jugement évaluant) ne répond pas à la théologie du Fils de Dieu montrée ci-dessus. 
L'idée de l'adoption apparaît uniquement dans la parabole de l'esclave très fidèle et non dans les explications concernant le Fils de Dieu. D'autant plus, il est inadmissible de caractériser l'enseignement d'Hermas comme un binitarisme parce que cette qualification provenant de la théologie ultérieure ne correspond pas au contenu de la foi exprimée dans le texte. Le schéma ultérieur d'interprétation de „pneuma-christologie” appliqué pour le Pasteur exige d'être précisé à la lumière de nos observations ci-dessus, ainsi il pourra être tenu pour un modèle d'interprétation au sens strict du terme.

Les résultats de nos recherches nous autorisent à constater que de nombreuses opinions diffusées sur la christologie de la Sim V sont des conceptions d'aspect, insuffisantes ou évidemment erronées, simplifiant le plus souvent la doctrine de cet écrit que nous pouvons appeler „la christologie” seulement dans le cas où elle serait dotée d'un commentaire précisant. Nous considérons que la détermination de l'enseignement d'Hermas comme une théologie judéochrétienne du Fils de Dieu est la plus juste et la plus précise. Et cette qualification, à notre avis, doit être appliquée au Pasteur entier. L'auteur de cet écrit s'appuie non seulement sur des idées doctrinales considérées déjà comme judéo-chrétiennes: la christologie angélomorfique, la pneuma-christologie, la nomos-christologie, l'onoma-christologie, mais il y puise d'une manière inventive pour sa propre vision théologique destinée en premier lieu aux judéochrétiens. Conscient du contexte culturel et religieux dans lequel vivait luimême et sa communauté, il développa un tel enseignement sur le Fils de Dieu qui pouvait être compris et accepté aussi par les Juifs et par les (pagano)chrétiens de cette époque-là. C'est cela que nous considérons comme le plus grand succès d'Hermas, qui pourrait constituer une inspiration importante dans le dialogue se déroulant actuellement entre les chrétiens et les Juifs. Dans ce domaine, F. Mussner a bien montré un problème extrêmement difficile: „Autant nous reconnaissons aujourd'hui à nouveau Jésus de Nazareth comme un Juif, autant nous savons aussi que la doctrine chrétienne sur Jésus, habituellement appelée „christologie”, qui sépare et probablement séparera toujours les chrétiens des Juifs. Avant tout, c'est la christologie de Fils de Dieu qui est pour le Juif la grande pierre d'achoppement dans la foi chrétienne" ${ }^{24}$. Nous ne partageons pas tout à fait cette opinion. De plus, le témoignage d'Hermas nous encourage à ce que les obstacles demeurant jusqu'ici dans la formulation courante de „la christologie de Fils de Dieu” soient surmontés. La proposition théologique formulée par Hermas pour ses coreligionnaires peut aussi intéresser et d'être d'une importance extraordinaire pour exprimer la foi en Fils de Dieu par les judéo-chrétiens d'aujourd'hui (Messianic Jews).

${ }^{24}$ F. Mussner, Traité sur les Juifs, trad. de l'allemand par R. Givord, Paris 1981, 363-364 (orig. Traktat über die Juden, München 1974, 336: Sohngotteschristologie). Le sujet est plus largement présenté par J.T. Pawlikowski, Christ in the Light of the Christian-Jewish Dialog, New York 1982. 


\section{TEOLOGIA JUDEOCHRZEŚCIJAŃSKA SYNA BOŻEGO \\ W PASTERZU HERMASA (SIM V 2,1 - 6,7A)}

(Streszczenie)

W licznych publikacjach poświęconych chrystologii Pasterza Hermasa centralne miejsce zajmuje Sim V. W dotychczasowych interpretacjach dominuje opinia, że Hermas jest przedstawicielem pneuma-chrystologii. Ponadto jego teologię charakteryzowano jako binitaryzm i adopcjonizm. Autor podejmuje interpretację Sim V na nowych podstawach, odwołując się do tradycji judeochrześcijańskiej. W rezultacie dokonanych analiz proponuje korektę wielu obiegowych opinii o tym piśmie i zawartej w niej teologii Syna Bożego. Z tej racji, że autor Pasterza świadomie wyeliminował ze swoich rozważań jakiekolwiek wątki mesjańskie i konsekwentnie nie posługuje się terminem „Chrystus”, ani jako tytułem, ani jako imieniem własnym, stąd też nie należy wprowadzać go do współczesnych interpretacji tego pisma. Okazuje się bowiem, że w bardzo licznych ,interpretacjach chrystologicznych” dostosowuje się w sposób nieuprawniony terminologię i treść Sim V do późniejszych kategorii teologicznych. Stąd autor postuluje, aby dochować wierności pierwotnym znaczeniom idei teologicznych zawartym w tekście. Wtedy też ujawnia się w pełni judeochrześcijański charakter teologii Syna Bożego w Sim V. 